\title{
Electro-orientation of a metal nanowire counterbalanced by thermal torques
}

\author{
Juan J. Arcenegui, ${ }^{1}$ Pablo García-Sánchez, ${ }^{1}$ Hywel Morgan, ${ }^{2}$ and Antonio Ramos ${ }^{1, *}$ \\ ${ }^{1}$ Departamento de Electrónica y Electromagnetismo, Facultad de Física, Universidad de Sevilla, Spain \\ ${ }^{2}$ School of Electronics and Computer Science, University of Southampton, United Kingdom
}

(Received 14 February 2014; published 16 June 2014)

\begin{abstract}
The rotational diffusion of electrically polarized metal nanowires suspended in an electrolyte is studied. The alignment of a Brownian nanowire in an ac field with a given direction is not complete due to thermal (fluctuating) torques. The orientation distribution allows us to examine the electrokinetic torques acting on the nanowire for smaller voltages than in previous deterministic experiments. In addition, the torques are obtained without recurring to the rotational friction coefficient as in dynamic deterministic experiments. The present results are in accordance with previous deterministic results of electro-orientation of metal nanowires. Nanowire rotation is originated by both the electrical torque on the induced dipole and by induced-charge electro-osmotic flow around the particle. At low frequencies of the applied ac field, induced-charge electro-osmotic orientation dominates while induced dipole torque orientation dominates at high frequencies. The angular standard deviation and the rotational rate are calculated from the measured fluctuating angle as a function of time, and good agreement with theoretical predictions is found. The experiments at high frequency indicate that the electrical torque on a nanowire near an insulating wall is reduced with respect to the bulk.
\end{abstract}

DOI: 10.1103/PhysRevE.89.062306

\section{INTRODUCTION}

Nanowires and nanotubes have attracted considerable attention in the research community for their potential use in biotechnology as biosensors [1], in microelectronics as building blocks of novel nanocircuits [2], or in solar energy technology for the absorption of light by means of a dense array of nanowires [3].

The manipulation and characterization of nanowires in solution using ac electric fields generated by microelectrodes is of particular interest because such technologies can easily be integrated into microdevices. To exploit such technologies it is important that the fundamental electrokinetic properties of these particles are fully understood. A metal particle suspended in an electrolyte and subjected to an electric field acquires an induced electrical double layer which dominates both the dielectric properties and the electrokinetic behavior of the particle at low frequencies. Metal particles suspended in electrolytes are often incorrectly modeled as lossy dielectrics characterized by a finite conductivity and permittivity ignoring the double-layer polarization at the metal particle-electrolyte interface [4-7]. This simplistic model predicts positive polarizabilities for all frequencies in the quasi-electrostatic regime and implies that there should not be any dipole relaxation for the metal particle at these frequencies. Furthermore, this model cannot explain the experimental observations of the electrorotation of metallic particles [8-11] or the frequency dependence of the electroorientation of metal rods [12].

Electro-orientation (EOr) describes the alignment of a nonspherical particle in an ac field along a fixed direction, while electrorotation (ROT) is the asynchronous rotation of a particle in a rotating electric field $[13,14]$. The EOr and ROT behavior of metal nanowires suspended in an electrolyte has been

*Corresponding author: ramos@us.es
PACS number(s): 47.57.jd, 81.07.Gf, 82.45.-h, 05.40.Jc

analyzed in previous publications [11,12]. Here we analyze the equilibrium EOr behavior of nanowires in competition with thermal (Brownian) forces. This experimental technique enables much lower voltages to be used than conventional experiments. A benefit is that the typical voltage across the double layer is then small and the more comprehensive electrokinetic theories are developed for voltages smaller than the thermal voltage $\left(k_{B} T / e=25 \mathrm{mV}\right)$. The metal nanowires have typical lengths of several microns and diameters of hundreds of nanometers. Although they quickly sediment because of their mass density, the nanowires do not rest on the bottom of the experimental chamber, but exhibit twodimensional Brownian motion at some small distance from the bottom. This happens in low conductivity electrolytes (as used in this work) where double layer repulsion prevents adhesion to the bottom wall. Under these conditions, the nanowires can orientate freely in an electric field. After application of the electric field the nanowires tend to align along the field direction, but Brownian motion leads to a distribution of angles around the field direction [15]. A measure of the electrokinetic torque acting on a particle is the external torque required to hold the particle at a certain orientation with respect to the applied field. In our experiments, the strength of the electrokinetic torque determines the amplitude of the nanowire angular fluctuations, and this provides an alternative method of measuring the EOr, particularly for smaller voltages. In the present work, we have been able to measure nanowire orientations for electric field amplitudes that were between 0.7 and 0.3 times smaller than the ones used in previous deterministic experiments. This means that we are able to study electrokinetic torques which are an order of magnitude smaller than previously (since they are quadratic with the electric field). Deterministic experiments of particle orientation (classical method) usually measure the particle rotational velocity, and from this the electrokinetic torque can be obtained by using the rotational friction coefficient. In the present case, the steady-state angular distribution is independent of the rotational friction coefficient. This parameter is difficult 
to measure accurately and depends on particle distance to the wall. In other words, the present experimental technique of balancing EOr against Brownian motion provides an independent way of measuring the electrokinetic torque acting on the particle, simply from a measurement of the angular distribution. ROT and EOr experiments of metal nanowires can be explained using dipole theory, with the assumption that the particles are perfectly polarizable (i.e., there are no Faradaic currents at the electrolyte-particle interface), and taking into account some correction from induced charge electro-osmosis (ICEO) theory $[11,12]$. In ac fields, perfectly polarizable metal particles have a characteristic frequency $\omega_{\mathrm{RC}}$ corresponding to the resistor-capacitor $(\mathrm{RC})$ circuit formed by the capacitance of the particle-electrolyte double layer and the resistance of the liquid bulk. As an example, the characteristic frequency for a sphere of radius $a$ is $\omega_{\mathrm{RC}}=2 \sigma /\left(C_{\mathrm{DL}} a\right)$ [9], where $\sigma$ is the conductivity of the electrolyte and $C_{\mathrm{DL}}$ is the double layer capacitance per unit area. For a cylinder, this characteristic frequency is a function of the aspect ratio. For frequencies of the applied electric field $\omega \ll \omega_{\mathrm{RC}}$, the induced double layer is fully charged, the electric field lines surround the particle, and it behaves as an insulator. For angular frequencies $\omega \gg \omega_{R C}$, there is negligible induced charge in the Debye layer, the electric field lines intersect perpendicularly with the particle surface, and the particle behaves as a perfect conductor. At low frequencies, when the nanowire behaves as an insulator, the induced dipole is negligibly small, as is the applied electrical torque on the particle. At high frequencies, when the nanowire behaves as a conductor, the induced dipole is maximum and the applied torque is able to orientate the nanowire. In addition to this, nanowire orientation is influenced by electroosmotic flow around the particle, i.e., ICEO orientation [15-18]. This flow occurs at low frequencies and is due to the interaction of the applied electric field with the induced charge in the double layer. Indeed it is the main mechanism of nanowire orientation at low frequencies, where the induced electrical dipole is negligibly small. Experimentally, the amplitude of ICEO orientation at low frequencies is several times smaller than dipole orientation at high frequencies [12]. The predicted ICEO orientation for an ideal double layer is much greater than that observed experimentally, which is in agreement with the observation that the ICEO slip velocity predicted for an ideal double layer is higher than that measured experimentally, sometimes by an order of magnitude [19]. Several reasons for this have been proposed including dielectric coating [20,21], counterion crowding [22], ion adsorption [21,23], surface roughness [24], surface conduction [25] and/or flow instability of the concentration polarization layer [26].

In this paper, the Brownian rotational motion of an electrically polarized metal nanowire is studied. Because the particles perform Brownian motion near the bottom of the experimental chamber, we assume in the analysis that nanowire motion is two dimensional, an important assumption that is validated experimentally. The background theory for the rotational diffusion of a metal Brownian nanowire subjected to an ac electric field is first described. Experimental observations and measurements of the EOr of silver nanowires are then presented. The angular standard deviation and the rotational rate are calculated from the measured fluctuating angles, and the data are discussed with respect to theory.

\section{THEORETICAL BACKGROUND}

\section{A. Summary of dipole and ICEO theories}

Slender-body theory together with the experimental results of EOr and ROT presented previously [12] show that particle rotation due to ICEO flow and particle rotation due to induced dipole torque (IDT) have the same characteristic frequency of the applied field, which we term $\omega_{\mathrm{RC}}$. In ROT, the IDT drives rotation in a counterfield direction, while ICEO drives rotation in a cofield direction. Both behaviors exhibit a single maximum at an angular frequency $\omega_{\mathrm{RC}}$. The nanowire angular velocity in ROT is of the form

$$
\dot{\theta}=f \frac{\varepsilon E_{0}^{2}}{\eta} \frac{\omega / \omega_{\mathrm{RC}}}{1+\left(\omega / \omega_{\mathrm{RC}}\right)^{2}},
$$

where $\eta$ and $\varepsilon$ are, respectively, the viscosity and dielectric permittivity of the electrolyte, $E_{0}$ is the amplitude of the rotating electric field, and $f$ is a nondimensional parameter that depends on the aspect ratio of the wire $\beta=b / a$ (here $a$ and $b$ are the nanowire semilength and radius, respectively). Both mechanisms result from the interaction of the out-of-phase induced charge with the applied electric field, which means that they both mirror the imaginary part of the particle polarizability. ROT experiments show counterfield rotation that closely matches dipole theory, indicating that the ICEO flow is much smaller than expected from ICEO theory for an ideal double layer.

In EOr, the two mechanisms occur over different frequency ranges, with an overlapping region around $\omega_{\mathrm{RC}}$. While ICEO driven rotation decreases with frequency, IDT rotation increases, so that the two spectra follow opposite trends. Both mechanisms tend to align the rod with the direction of the applied electric field. When subjected to an applied electric field with fixed direction, the nanowire angular velocity is of the form

$$
\dot{\theta}=f \frac{\varepsilon E_{0}^{2}}{\eta}\left(\frac{\Lambda}{1+\left(\omega / \omega_{\mathrm{RC}}\right)^{2}}+\frac{\left(\omega / \omega_{\mathrm{RC}}\right)^{2}}{1+\left(\omega / \omega_{\mathrm{RC}}\right)^{2}}\right) \sin \theta \cos \theta
$$

where $\theta$ is the angle between the nanowire long axis and the direction of the applied electric field. Here $\Lambda$ is a nondimensional parameter that accounts for the different surface effects that reduce the slip velocity in a nonideal double layer: surface roughness, ion adsorption and/or dielectric coating. In EOr, both IDT and ICEO result from the interaction of the in-phase induced charge with the applied electric field, which means that they both mirror the real part of the particle polarizability. The measured characteristic angular speed due to IDT is much greater than that due to ICEO (experimentally $\Lambda \ll 1$ ), which indicates again that ICEO flow is much smaller than expected from standard theory.

\section{B. Fokker-Planck formulation for orientation distribution}

Because of gravity, the nanowires sink and fluctuate on the bottom surface of the experimental device, undergoing translational and rotational Brownian motion in two dimensions (they stay within the focal plane of the microscope). Experimentally, the nanowires orient almost parallel to the bottom, i.e., their polar angle fluctuates although they remain very close to the 
horizontal direction. In addition, their center-of-mass position fluctuates near the wall. The objective of this work is to study the orientation of the nanowires within the focal plane. As a first approximation, it is assumed that the rotational diffusion is characterized by a single diffusion coefficient $D_{\theta}$. Since the nanowires stay within the focal plane near the bottom, this system is similar to previous works characterizing the rotational diffusion of protein filaments [27] or ellipsoids [28] confined between two walls.

Deterministic alignment due to both ICEO flow around the particle and the electrical torque on the induced dipole is counterbalanced by Brownian (fluctuating) torques. The probability density function (PDF) that describes the orientation distribution is governed by the Fokker-Planck equation in one dimension [15,29]

$$
\frac{\partial \Psi}{\partial t}+\frac{\partial}{\partial \theta}\left(\Psi \dot{\theta}_{\operatorname{det}}-D_{\theta} \frac{\partial \Psi}{\partial \theta}\right)=0,
$$

where $\dot{\theta}_{\text {det }}$ is the deterministic angular speed, which in our case has the form [12]

$$
\dot{\theta}_{\mathrm{det}}=-\Gamma \sin (2 \theta)
$$

and $D_{\theta}$ is the coefficient of rotational diffusion. Here the orientational rate $\Gamma$ is a parameter that depends on the frequency of the applied electric field and is proportional to $\varepsilon E_{0}^{2} / \eta$ [Eq. (2)]. The Einstein relation for the rotational diffusion coefficient is $D_{\theta}=k_{B} T / \gamma$, where $\gamma$ is the viscous coefficient for rotational motion perpendicular to the primary axis. In the experiments in this paper, this rotational diffusion coefficient is understood to be an average parameter that accounts for the variation in the viscous drag with height and polar angle that the nanowire explores in its fluctuations [27].

The steady-state solution to Eq. (3) that satisfies the condition of even symmetry $\partial \Psi / \partial \theta=0$ at $\theta=0$ is

$$
\Psi=\Psi_{0} e^{-\frac{\Gamma \sin ^{2}(\theta)}{D_{\theta}}},
$$

where $\Psi_{0}$ is obtained from the normalization condition $\int_{-\pi / 2}^{\pi / 2} \Psi d \theta=1$. Here we have restricted the angle domain to $(-\pi / 2, \pi / 2)$ because for a cylinder, angles $\theta=\pi-\alpha$ and $\theta=-\alpha$ are indistinguishable. For $\Gamma / D_{\theta} \gg 1$, the PDF is negligibly small except for $\theta \ll 1$, so that the PDF approaches a Gaussian distribution

$$
\Psi \approx \frac{\Gamma}{\pi D_{\theta}} e^{-\frac{\Gamma}{D_{\theta}} \theta^{2}} .
$$

At high signal frequencies, there is negligible ICEO flow and the orientation is due entirely to the torque on the induced charge on the particle surface. If the particle is far from a wall, this torque can be written as the torque on the induced dipole. In this case, the deterministic angular speed is [12]

$$
\dot{\theta}_{\text {det }}=\frac{\tau_{e}}{\gamma}=-\frac{E_{0}^{2}}{4 \gamma} \operatorname{Re}\left[\alpha_{x}-\alpha_{y}\right] \sin (2 \theta),
$$

where $\tau_{e}$ is the electrical torque on the particle, $\alpha_{x}$ and $\alpha_{y}$ are, respectively, the polarizability along the particle long axis and along the short axis. The minus sign indicates that the torque tends to reduce the angle $\theta$ if $\operatorname{Re}\left[\alpha_{x}-\alpha_{y}\right]>0$. For slender cylinders $\left|\alpha_{x}\right| \gg\left|\alpha_{y}\right|$, which means that $\alpha_{y}$ can be neglected in the following analysis. The potential energy associated with the torque $\tau_{e}$ is

$$
U=\frac{1}{4} \operatorname{Re}\left[\alpha_{x}\right] E_{0}^{2} \sin ^{2} \theta .
$$

From statistical mechanics, the probability density function of the equilibrium state is given by the Boltzmann factor

$$
\Psi(\theta)=\Psi_{0} \exp \left[-\frac{U}{k_{B} T}\right]=\Psi_{0} \exp \left[-\frac{\operatorname{Re}\left[\alpha_{x}\right] E_{0}^{2} \sin ^{2} \theta}{4 k_{B} T}\right],
$$

which is equivalent to Eq. (5). When the nanowire is close to an insulating wall, the torque exerted by the applied field is different from that seen in the bulk. The torque near the wall is expected to be smaller than in the bulk (see Sec. IV). Equation (9) can still be used in terms of an effective polarizability, which is also a function of the distance to the wall.

\section{Autocorrelation function}

When the angular dispersion is small, Eq. (4) can be linearized and the PDF becomes

$$
\frac{\partial \Psi}{\partial t}-2 \Gamma \frac{\partial}{\partial \theta}(\theta \Psi)-D_{\theta} \frac{\partial^{2} \Psi}{\partial \theta^{2}}=0 .
$$

This equation describes the Brownian motion of an overdamped harmonic oscillator. The autocorrelation function is then $[30,31]$

$$
\langle\theta(t+\tau) \theta(t)\rangle=\left\langle\theta^{2}(t)\right\rangle e^{-2 \Gamma \tau}=\frac{k_{B} T}{K} e^{-2 \Gamma \tau},
$$

where $K=2 \Gamma / D_{\theta}$ is the constant of the harmonic oscillator and $\left\langle\theta^{2}(t)\right\rangle=k_{B} T / K$ comes from the equipartition theorem. In our case, and for the limit of high frequency $K=$ $\frac{1}{2} \operatorname{Re}\left[\alpha_{x}\right] E_{0}^{2}$. For data analysis, the mean square angular displacement (MSD) will be used $\operatorname{MSD}_{\theta}=\left\langle(\theta(t+\tau)-\theta(t))^{2}\right\rangle$, which is related to the autocorrelation function and has the form [32]

$\operatorname{MSD}_{\theta}(\tau)=2\left\langle\theta^{2}\right\rangle-2\langle\theta(t+\tau) \theta(t)\rangle=\frac{2 k_{B} T}{K}\left(1-e^{-2 \Gamma \tau}\right)$.

Therefore, measurements of the angular fluctuation of the nanowire in a thermal bath provide information of the rate of orientation $\Gamma$ [32], which can be directly compared to the measurements of $\Gamma$ from other deterministic experiments [12].

\section{EXPERIMENTS}

\section{A. Experimental details}

The silver nanowires were supplied by "Nanostructured \& Amorphous Materials, Inc" and are provided as a paste consisting of $70 \mathrm{wt} \%$ silver and $30 \mathrm{wt} \%$ ethanol. Polyvinylpyrrolidone (PVP) is added during synthesis of the nanowires, and might be present on the silver surface. Figure 1(a) is a scanning electron microscope (SEM) image of the nanowires; with dispersed length $(2 a)$ and width $(2 b)$. The diameter $(2 b)$ of the wires varies between 160 and $320 \mathrm{~nm}$. In this work, we measured the properties of nanowires with lengths ranging between six and seven microns. A statistical analysis of the aspect ratio 

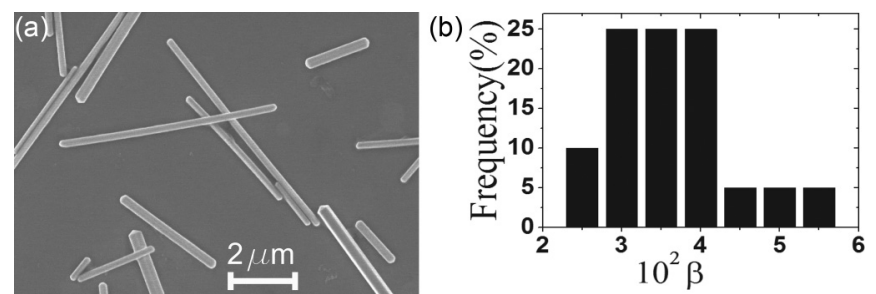

FIG. 1. (a) SEM image of silver nanowires. (b) The histogram is the result of the statistical study of the nanowires aspect ratio. Wires aspect ratio varies between 0.03 and 0.05 .

( $\beta=b / a$ ) of the nanowires based on 21 different SEM images is shown in Fig. 1(b). The mean value of the aspect ratio was $\beta=0.04 \pm 0.01$, where the uncertainty is the standard deviation of the distribution.

Prior to use, the nanowires were washed several times in an aqueous solution of $\mathrm{KCl}$ with a specific concentration. The experiments were performed with three values of electrolyte conductivity: $1.5,5$, and $15 \mathrm{mS} / \mathrm{m}$. After washing, a suspension of nanowires was placed at the center of an electrode array made of four circularly shaped planar microelectrodes, and the appropriate ac signal was applied [see Fig. 2(a)]. The total potential difference between electrodes was $V(t)=V_{0} \cos (\omega t)$. The microelectrodes were made using photolithography and consisted of a 300-nm-thick platinum layer patterned on a glass substrate. The diagonal gap between opposite electrodes was $0.5 \mathrm{~mm}$. For the EOr experiments the electrical connections are as shown in Fig. 2(a). This generated an ac electric field $(E)$ of fixed direction in the center of the electrode array. When a silver nanowire was placed in the center it orientated along this axis. However, because of Brownian motion the nanowire orientation is not complete and there is a dispersion in the angle $\theta$ between the axis of the wire and the electric field [see Fig. 2(b)]. This experimental angular distribution is bell-shaped, centered along the direction of the applied electric field. All experiments were observed using an inverted microscope and videos of nanowire orientation were analyzed using a custom-made program in MATLAB.

\section{B. Experimental results}

Figure 3 shows the distribution of angles for three different voltage amplitudes, for low [Fig. 3(a)] and high [Fig. 3(b)] frequencies of an applied signal. The number of events in these

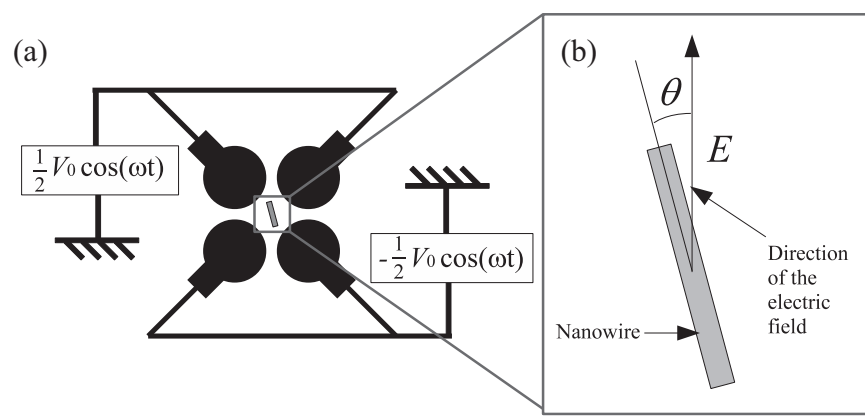

FIG. 2. (a) Electrical connections for EOr experiments. (b) Angle $\theta$ between the applied electric field and the long axis of the nanowire. histograms is around 1800 and the electrolyte conductivity is $1.5 \mathrm{mS} / \mathrm{m}$. As the voltage is increased the angle distribution narrows, i.e., the nanowires become more oriented. In addition, orientation at high frequency $(800 \mathrm{kHz})$ is greater than at low frequency $(3.2 \mathrm{kHz})$. Note the different scales for the abscissa in the two graphs. At this low conductivity, the nanowire behaves as an insulator at a frequency of $3.2 \mathrm{kHz}$ and as a conductor at a frequency of $800 \mathrm{kHz}[11,12]$. Typical frequencies for transition (i.e., maximum of ROT as given in our previous publications) are $40 \mathrm{kHz}$ for $1.5 \mathrm{mS} / \mathrm{m}$, $100 \mathrm{kHz}$ for $5 \mathrm{mS} / \mathrm{m}$, and $200 \mathrm{kHz}$ for $15 \mathrm{mS} / \mathrm{m}$. Therefore, for an electrolyte of conductivity $1.5 \mathrm{mS} / \mathrm{m}, 3.2 \mathrm{kHz}$ is representative of a low frequency and $800 \mathrm{kHz}$ representative of a high frequency. We have previously shown [12] that at low frequencies, nanowire orientation is mainly driven by ICEO flow, while at high frequencies nanowire orientation is mainly due to the electrical torque on the induced dipole. The figure shows that orientation due to ICEO (low frequency) is much weaker than orientation due to the torque on the induced dipole (high frequency) for the same voltage amplitude, in agreement with our previous observations [12]. Furthermore, these experimental angle distributions follow the expected trend given by Eq. (5).

Since the typical measured angles $\theta$ are small, $\sin \theta \approx \theta$ and the PDF becomes a normal distribution [Eq. (6)]

$$
\Psi(\theta)=\frac{1}{\sigma \sqrt{2 \pi}} e^{-\frac{\theta^{2}}{2 \sigma^{2}}} .
$$

Figure 4 shows the standard deviation $\sigma$ as a function of frequency for three electrolyte conductivities. The standard deviation was obtained by fitting the experimentally determined angular distribution to a Gaussian distribution. For some values of $\sigma$ we checked that the $\sigma$ values obtained by fitting to the original PDF $\Psi=\Psi_{0} \exp \left[-\left(\sin ^{2} \theta / 2 \sigma^{2}\right)\right]$ were in agreement with those obtained from a fit to the Gaussian. The standard deviation $\sigma$ as a function of frequency shows that orientation is greater at high frequency than at low frequency for the same applied voltage. It also shows that $\sigma$ at high frequency is the same for all three conductivities, while at low frequency $\sigma$ increases with conductivity, i.e., nanowire orientation decreases with conductivity at low frequencies. These features of nanowire orientation shown in the figure agree with our previous publication [12], namely: (a) EOr at high frequency is greater than EOr at low frequency, and (b) EOr at high frequencies does not depend on electrolyte conductivity while at low frequencies it decreases with conductivity. In particular, this later observation is a manifestation of the fact that ICEO flow around the particle (the mechanism of orientation at low frequencies) decreases with conductivity, as observed in ICEO experiments $[19,33,34]$. For quantitative comparison to previous results we need to examine the relationship between the standard deviation $\sigma$ and the orientational rate $\Gamma$, the parameter that is measured directly in the deterministic EOr experiments. According to Eq. (6) the relationship is

$$
\sigma^{2}=\frac{D_{\theta}}{2 \Gamma}=\frac{k_{B} T}{2 \gamma \Gamma} .
$$

The theoretical viscous coefficient $\gamma$ for a cylinder (away from a wall) with aspect ratio $\beta=0.04$ is given in [35]. Converting the value of $\Gamma$ to the value expected from the 


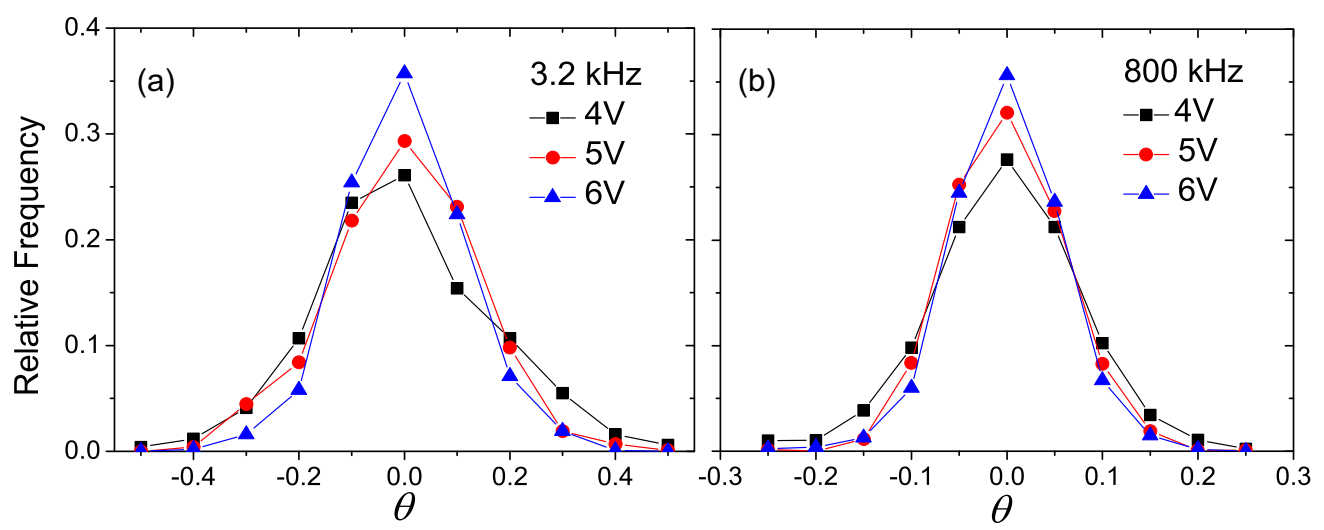

FIG. 3. (Color online) Distribution of angles as function of voltage amplitude $V_{0}$ for a frequency of (a) 3.2 and (b) $800 \mathrm{kHz}$. The dispersion of angles decreases as the voltage increases. Note the different scales on the abscissa axis in both figures showing that the angle distribution is narrower for higher frequency.

present experiments (assuming $\Gamma \propto E_{0}^{2}$ ), then the difference between the experimental values of $\sigma$ and the values expected from previous experiments is less than $20 \%$ for the low frequency limit, and less than $10 \%$ for the high frequency limit.

Both ICEO orientation and dipole torque orientation are proportional to the applied electric field squared. This means that the standard deviation $\sigma$ should be inversely proportional to voltage. Figure 5 shows $\sigma$ as a function of reciprocal voltage for two values of frequency, 3.2 and $800 \mathrm{kHz}$ (for an electrolyte conductivity of $1.5 \mathrm{mS} / \mathrm{m}$ ). As in Fig. 4, particle orientation is greater at high frequency (smaller standard deviation $\sigma$ ). The straight dashed line is the theoretical result for an induced dipole where the particle behaves as a conductor, and is far from the wall. At high frequencies nanowire orientation is due to the electrical torque on the induced dipole and the PDF is proportional to the Boltzmann factor, Eq. (9). Therefore, the relationship between $\sigma$ and the polarizability $\alpha_{x}$ is

$$
\sigma=\sqrt{\frac{2 k_{B} T}{\operatorname{Re}\left[\alpha_{x}\right] E_{0}^{2}}},
$$

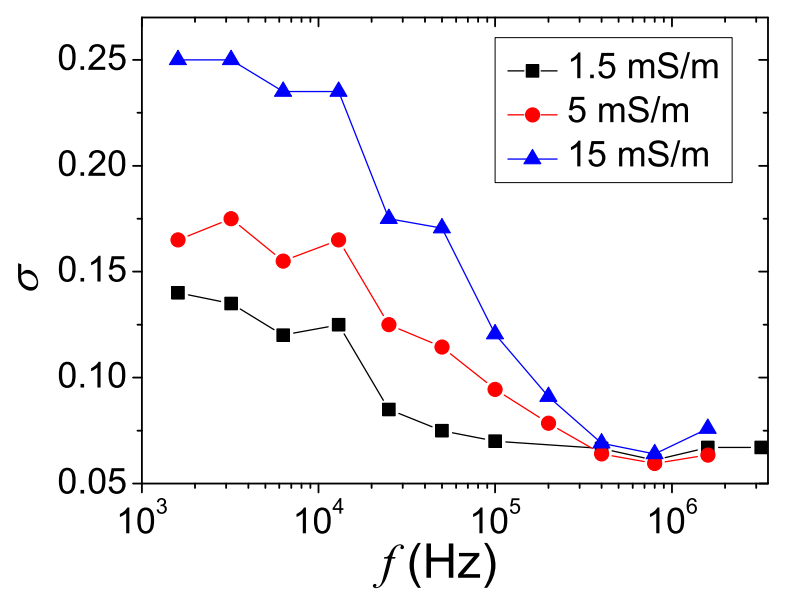

FIG. 4. (Color online) Standard deviation $\sigma$ as function of frequency for three conductivities: $1.5,5$, and $15 \mathrm{mS} / \mathrm{m}$. The applied voltage amplitude was $V_{0}=5 \mathrm{~V}$. giving the theoretical straight line shown in the figure. The polarizability is given by $\alpha_{x}=4 \pi \varepsilon a^{3} A$, where $A$ is nondimensional. For the previous calculations $k_{B} T=4.14 \times$ $10^{-21} \mathrm{~J}$, with $T=300 \mathrm{~K}$, nanowire half-length $a=3.25 \mu \mathrm{m}$, nondimensional polarizability $\operatorname{Re}[A]=0.155$ for aspect ratio $b / a=0.04$. The electric field was estimated to be $E_{0}^{2}=$ $2.37 V_{0}^{2} \times 10^{6} \mathrm{~V}^{2} \mathrm{~m}^{-2}$, calculated numerically from the average torque within the field of view. To obtain this torque, Laplace's equation for the electrical potential was solved using the commercial program COMSOL. The ratio between the experimental and theoretical values of $\sigma$ is $1.1 \pm 0.1$ for the data in Fig. 5 (electrolyte conductivity of $1.5 \mathrm{mS} / \mathrm{m}$ ). The same ratio for $\sigma$ at high frequency (the data from Fig. 4), using the three conductivities, gives $\sigma^{\exp } / \sigma^{\text {theo }}=1.19 \pm 0.08$. These results indicate that the predicted torque in the bulk is higher than that measured near the wall.

For high frequencies, and according to Eq. (15), $\sigma E_{0}$ is a function of half-length $a$ and is independent of $E_{0}$. Figure 6 shows a $\log -\log$ plot of $\sigma V_{0}$ as a function of nanowire length for a conductivity of $1.5 \mathrm{mS} / \mathrm{m}$, and a frequency of $800 \mathrm{kHz}$. This figure shows the dependence of $\sigma$ on nanowire length, (which

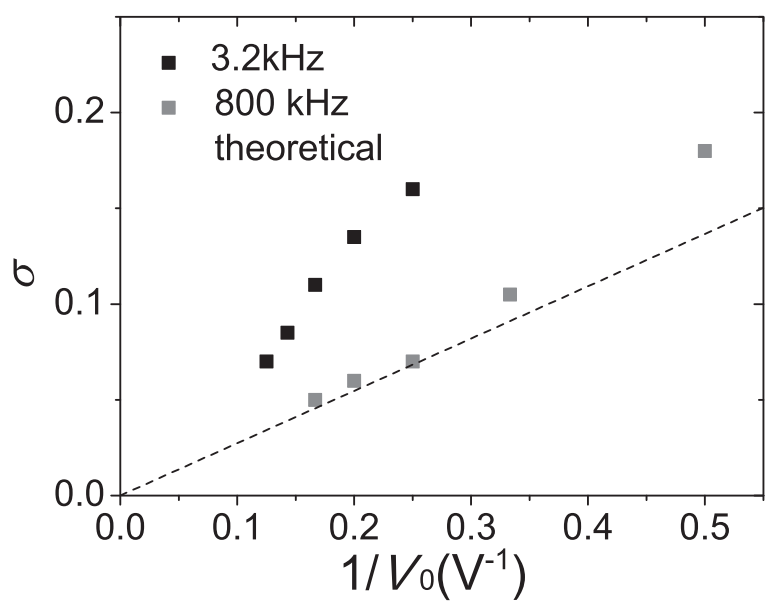

FIG. 5. Standard deviation $\sigma$ as function of reciprocal of voltage $1 / V_{0}$ for two fixed values of frequency: 3.2 and $800 \mathrm{kHz}$. The dashed line is theoretical, valid for high frequency. The liquid conductivity is $1.5 \mathrm{mS} / \mathrm{m}$. 


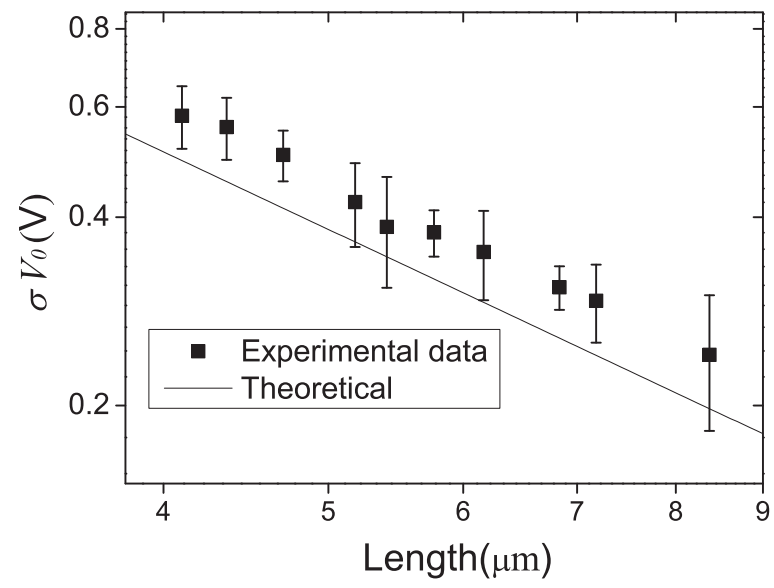

FIG. 6. Standard deviation $\sigma$ as function of nanowire length. Case for high frequency $(800 \mathrm{kHz})$ and an electrolyte conductivity of $1.5 \mathrm{mS} / \mathrm{m}$.

for the other figures of this work was set to be in the range between 6 and $7 \mu \mathrm{m}$ ). The experimental measurements were obtained using different voltage amplitudes $V_{0}$ between 2 and $6 \mathrm{~V}$. According to Eq. (15), the figure is the standard deviation for $V_{0}=1 \mathrm{~V}$ as a function of nanowire length. For this plot, the angle standard deviation $\sigma$ was computed using the square root of the average of the squares $\left(\sqrt{\sum_{i} \theta\left(t_{i}\right)^{2} / N}\right)$ following individual particles in time (instead of fitting the angular dispersion to a Gaussian). Measurements were performed for ten particles of a given length, and the error bars are the dispersions of these measurements. The theoretical curve is obtained from Eq. (15), which is valid for high frequency. The angular standard deviation $\sigma$ is proportional to $a^{-3 / 2} \operatorname{Re}[A]^{-1 / 2}$, where the nondimensional polarizability $\operatorname{Re}[A]$ varies slowly with $a$. The experimental values of $\sigma \times V_{0}$ follow the trend given by Eq. (15) although they are all systematically greater. This was also true for the results in Figs. 4 and 5. For the results in Fig. 6, the ratio between experimental and theoretical values of $\sigma$ is $\sigma^{\exp } / \sigma^{\text {theo }}=1.2 \pm 0.2$.

We investigated the angular dynamics of the nanowires to determine the rotational rate $\Gamma$. Figure 7 shows an example of

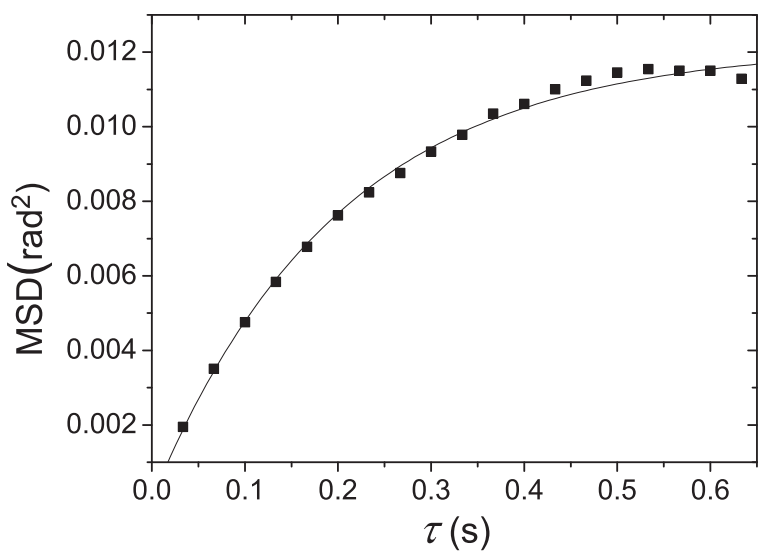

FIG. 7. $\mathrm{MSD}_{\theta}$ as a function of $\tau$. Applied signal of $V_{0}=6 \mathrm{~V}$, $f=800 \mathrm{kHz}$, electrolyte conductivity $1.5 \mathrm{mS} / \mathrm{m}$, nanowire length $=$ $6.5 \mu \mathrm{m}$.

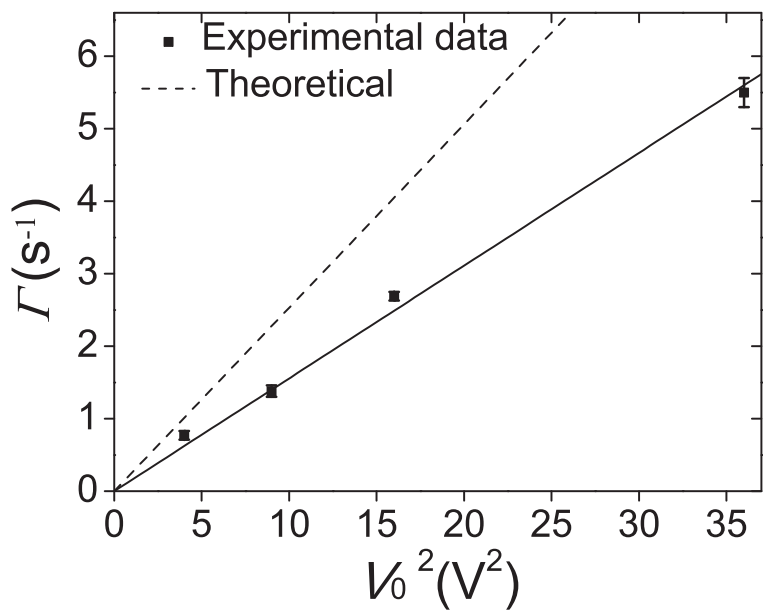

FIG. 8 . $\Gamma$ as a function of voltage amplitude squared $V_{0}^{2}$. Case for $f=800 \mathrm{kHz}$, conductivity $1.5 \mathrm{mS} / \mathrm{m}$, length $6.5 \mu \mathrm{m}$.

the mean square angular displacement $\operatorname{MSD}_{\theta}=\langle(\theta(t+\tau)-$ $\left.\theta(t))^{2}\right\rangle$ as a function of time lag $\tau$ for the high frequency case. The $\operatorname{MSD}_{\theta}(\tau)$ curve was obtained from the angular trajectory of a nanowire and this was averaged for four particles. More than 300 angles were measured for each selected nanowire. From the fit to the theoretical expression for $\mathrm{MSD}_{\theta}$, Eq. (12), the exponential factor $2 \Gamma$ is obtained. The figure shows a correlation time of around $0.2 \mathrm{~s}$ and the $\mathrm{MSD}_{\theta}$ curve saturates beyond $0.4 \mathrm{~s}$, indicating that correlation is lost for that time increment.

Figure 8 shows the values of $\Gamma$ obtained from the fit of $\operatorname{MSD}_{\theta}$ to Eq. (12). It depicts $\Gamma$ as a function of voltage amplitude squared $V_{0}^{2}$ for the high frequency case. The plot also shows the prediction for $\Gamma$ if the nanowires were in the liquid bulk (here we have used the viscous friction coefficient corresponding to $\beta=0.04$ given in [35]). The ratio between the experimental and theoretical values is $\Gamma^{\exp } / \Gamma^{\text {theo }}=0.62 \pm$ 0.04 . The orientational rate $\Gamma$ was obtained by deterministic experiments in our previous work [12]. Using the data from this previous work, the ratio $\Gamma^{\exp } / \Gamma^{\text {theo }}=0.74 \pm 0.07 .{ }^{1}$ The results from the two methods are close to each other and both are smaller than the theoretical prediction for the liquid bulk.

\section{DISCUSSION AND CONCLUSION}

The Brownian stochastic EOr experiments are in qualitative agreement with previous deterministic EOr experiments. Thus, the angle dispersion at equilibrium has shown that low frequency EOr is much weaker than high frequency EOr for the same applied electric field. With respect to a quantitative comparison to previous experiments, the standard deviation in angle predicted using previous experimental parameters is in agreement with the observed angular standard deviation. A quantitative comparison to the theory is difficult for low

\footnotetext{
${ }^{1}$ This value differs slightly from that given in [12] because it is calculated using the rotational friction coefficient given in [35], which seems to be more accurate than that given in [36] for our nanowire slenderness.
} 
frequency experiments because ICEO depends strongly on the characteristics of the particle surface, which are not well controlled. It is observed that EOr at low frequencies is much smaller than predicted for an ideal double layer, i.e., without taking into account effects like surface roughness [24], ion adsorption [21,23], or dielectric coating [20,21]. Other effects like ion crowding [22], surface conduction [25], and flow instability of the concentration polarization layer [26] may be neglected in our experiments because the typical voltage across the double layer (given by typical electric field times nanowire half-length $E_{0} a$ ) was close to $25 \mathrm{mV}$ or smaller. It is also observed that EOr at low frequencies decreases with electrolyte conductivity, which mirrors the experimental observation that ICEO decreases as electrolyte conductivity increases. A quantitative comparison to the theory is possible in the high frequency limit where the deterministic torque arises entirely from the electrical torque on the induced dipole. Here good agreement is found: the angular standard deviation $\sigma$ as a function of voltage (Fig. 5), $\sigma$ as a function of length (Fig. 6), as well as the orientational rate $\Gamma$ as a function of voltage (Fig. 8) follow expected trends.

The angular standard deviation $\sigma$ shown in Figs. 3 to 6 is a feature of the equilibrium state, where the dynamics required to reach equilibrium are not present. In other words, this parameter is independent of the rotational friction coefficient [see Eq. (15)]. The angular standard deviation from IDT deviates from prediction by between $10 \%$ to $20 \%$. Most of this discrepancy can be ascribed to the influence of the wall on the electrical torque. If the electrical torque is reduced by a factor $\tau_{e}^{\text {wall }} / \tau_{e}^{\text {bulk }}$ between 0.69 and 0.83 , the measured angular standard deviation would conform to theory (note that $\sigma^{2} \propto$ $\left.1 / \tau_{e}\right)$. In our previous paper we ascribed most of the difference between dipole theory and high frequency experiments to wall effects on the viscous torque. In view of the present experiments, wall effects should also be important for the electrical torque. Qualitatively the electrical torque should be reduced due to the presence of the wall as follows. The electric field that surrounds a polarizable particle near a wall can be seen as the sum of the applied electric field plus the electric field that the image charges create on the particle. For an insulating wall (where the boundary condition for the potential is normal current equal to zero) the image charges do not change sign and, therefore the image of a dipole placed at $z=h$ is an identical dipole placed at $z=-h$ (see Fig. 9). The dipole image creates an electric field on the particle in the direction opposite to the applied field, which in turn reduces the overall electric field. The Appendix shows numerical calculations of the electrical torque for the high frequency limit. The numerical calculations were performed using finite element analysis for the complete electrical problem and also using numerical calculations for a slender-body approximation. The results indicate that the electrical torque can be reduced by up to a factor of 0.6 with respect to the torque in the bulk.

Figures 7 and 8 show the rate of orientation $\Gamma$ obtained from the mean square angular displacement as a function of increment of time. These values of $\Gamma$ can be compared to the values obtained from deterministic experiments [12]. Comparing just the values for the high frequency limit shows that the experimental values of $\Gamma$ from the two methods are close; the Brownian experiments have values around $20 \%$

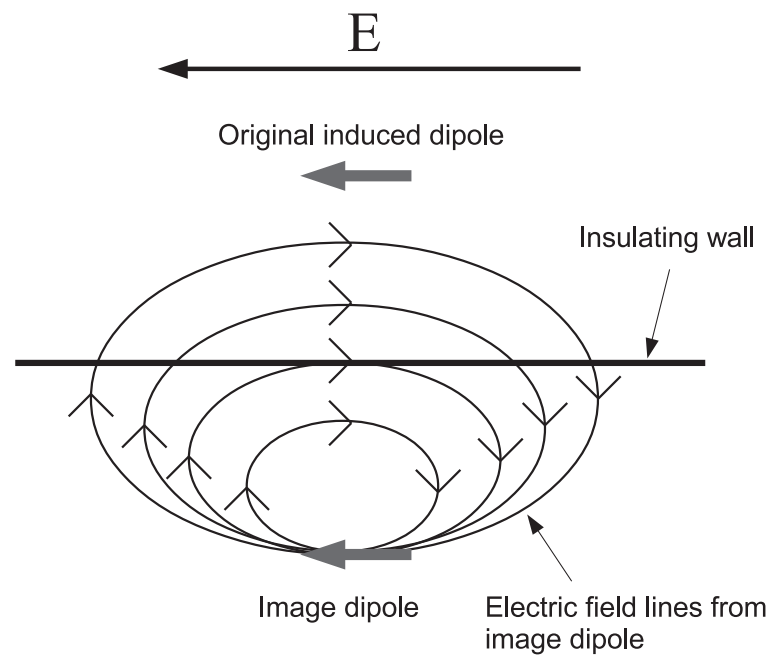

FIG. 9. Induced dipole parallel to an insulating wall and its image dipole.

smaller. An important difference between the two methods is that the present experiments were performed in a lower voltage range. This discrepancy might indicate a voltage dependence of nanowire equilibrium distance to the wall. However, the dispersion in the measurements is too high to be conclusive, and it would be interesting to pursue this issue further. Both methods provide $\Gamma$ values smaller than the theoretical IDT prediction for the bulk. In the present experiments $\Gamma^{\exp } / \Gamma^{\text {theo }}=0.62 \pm 0.04$ and part of the reduction can be explained by a reduction of the electrical torque $\tau_{e}^{\text {wall }} / \tau_{e}^{\text {bulk }}$ between 0.69 and 0.83 , but the increase of viscous friction near wall is also needed to conform experiments with theory. Therefore, the proximity to the insulating wall affects the electrical torque as well as the viscous torque, and both effects contribute to a reduction in the angular velocity (the electrical torque diminishes and the viscous torque augments).

To conclude, experiments of the electro-orientation of nanowires counterbalanced by stochastic thermal torques have been performed. The nanowires have a high mass density and undergo Brownian motion close to the bottom wall of the experimental system, where they are oriented in an ac electric field. This method has allowed us to investigate the electroorientation of metal nanowires for a smaller voltage range than previously used in deterministic experiments. The results are in agreement with previous experimental results: nanowire rotation is driven by both the electrical torque on the induced dipole and by induced-charge electro-osmotic flow around the particle. At low frequencies, induced-charge electro-osmotic rotation dominates while induced dipole torque rotation dominates at high frequencies. Good agreement with theoretical predictions is found in the high frequency limit, which confirms the validity of the method. The experiments at high frequency suggest that there is a reduction in the electrical torque on the nanowire near an insulating wall.

\section{ACKNOWLEDGMENTS}

We acknowledge financial support from Junta de Andalucía (Contract No. P09-FQM-4584) and the Spanish Government Ministry MEC (Contract No. FIS2011-25161). 


\section{APPENDIX}

In this Appendix we analyze the effect of an insulating wall on the electrical torque on a conducting cylinder. The insulating wall forms the plane $z=0$ and the cylinder long axis is parallel to the wall and along the $x$ direction, placed at $z=h$. An electric field is applied to the cylinder at an angle of $45^{\circ}$ with respect to the cylinder axis, $\mathbf{E}_{0}=\frac{1}{\sqrt{2}} E_{0}\left(\mathbf{u}_{x}+\mathbf{u}_{y}\right)$. Near a wall, the electrical torque cannot be calculated simply as the torque on an induced dipole. The electric field that acts on the particle is sum of the externally applied field plus that due to image charges. In this Appendix the electrical torque is computed numerically by two methods. The first method solves Laplace's equation for the electrical potential in three dimensions $\nabla^{2} \Phi=0$ using the finite element method, and the Maxwell stresses are integrated over the surface of the cylinder

$$
\tau_{z}=\mathbf{u}_{z} \cdot \int \mathbf{r} \times \mathrm{T}_{e} \cdot d \mathbf{S},
$$

where $\tau_{z}$ is the electrical torque around the vertical axis $z$ and $\mathrm{T}_{e}=\varepsilon \mathbf{E E}-\frac{1}{2} \varepsilon E^{2} \mathrm{I}$ is the Maxwell stress tensor. The boundary conditions for the potential are: (a) normal derivative equal zero $\partial \Phi / \partial n=0$ at the insulating wall; (b) fixed potential on the cylinder surface equal to zero $\Phi=0$; and (c) fixed potential far from the cylinder corresponding to the applied electric field, $\Phi=-\frac{1}{\sqrt{2}} E_{0}(x+y)$. The electrical potential and the electrical torque were computed using finite elements with the commercial program COMSOL.

In the second method, numerical calculations of the torque are performed using a slender-body approach, valid for distances to wall much greater than the cylinder radius $(h \gg b)$. In the slender-body approximation, the electrical potential due to the charges induced on the surface of the conducting particle can be written as if this potential were created by an effective linear charge density $\lambda$ distributed along the particle axis [37-39]. Taking into account the image charges due to the insulating wall, the potential outside the slender cylinder can be written as

$$
\begin{aligned}
\Phi(\mathbf{r})= & -\mathbf{E}_{0} \cdot \mathbf{r}+\frac{1}{4 \pi \varepsilon} \int_{-a}^{a} \frac{\lambda(s)}{\left|\mathbf{r}-\mathbf{r}_{1}(s)\right|} d s \\
& +\frac{1}{4 \pi \varepsilon} \int_{-a}^{a} \frac{\lambda^{\prime}(s)}{\left|\mathbf{r}-\mathbf{r}_{2}(s)\right|} d s,
\end{aligned}
$$

where $\mathbf{E}_{0}$ is the applied field, $\mathbf{r}_{1}(s)=(s, 0, h)$ is the position vector of the linear charge (which is the particle axis), $\lambda^{\prime}(s)=$ $\lambda(s)$ for an insulating wall, and $\mathbf{r}_{2}(s)=(s, 0,-h)$ is the position vector of the linear image charge. In the slender-body approximation, the electrical torque comes from the action of

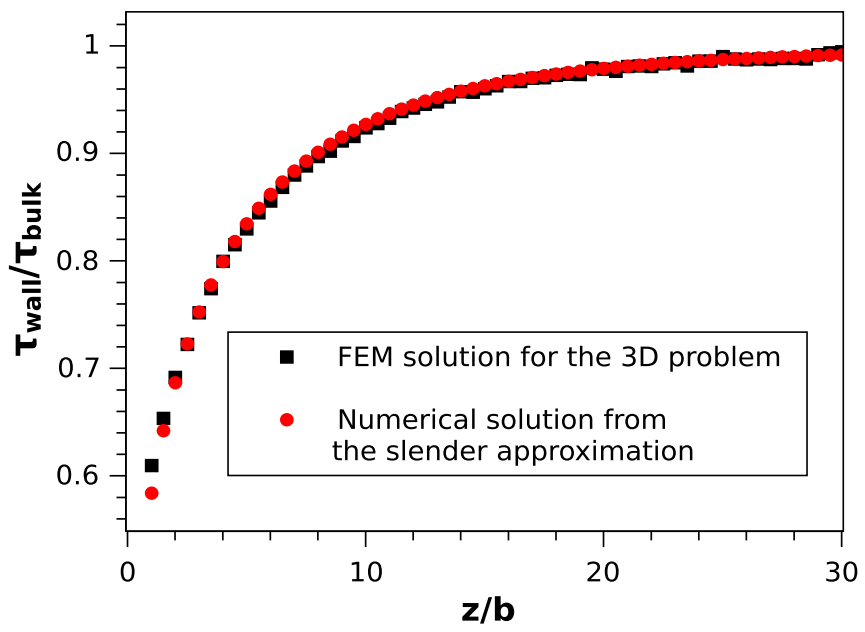

FIG. 10. (Color online) Electrical torque near wall on a cylinder $\tau_{\text {wall }}$ normalized with the one in the bulk $\tau_{\text {bulk }}$ as a function of nondimensional distance to the wall $h / b$. Case $b / a=0.04$.

the electric field perpendicular to the axis $\mathbf{E}_{0 y}$ on the induced charge due to the electric field parallel to the axis $\mathbf{E}_{0 x}$ [12]. Therefore, we need only to calculate $\lambda$ induced by $\mathbf{E}_{0 x}$. The integral equation that determines $\lambda$ comes from imposing that the potential is zero on the cylinder surface

$$
\begin{aligned}
4 \pi \varepsilon E_{0 x} x= & \int_{-a}^{a} \frac{\lambda(s)}{\sqrt{(x-s)^{2}+b^{2}}} d s \\
& +\int_{-a}^{a} \frac{\lambda(s)}{\sqrt{(x-s)^{2}+(2 h)^{2}}} d s,
\end{aligned}
$$

where the potential due to the image charge on the cylinder surface has been approximated taking into account $(2 h \gg b)$. The integral equation (A3) is solved numerically using the Galerkin method. The linear charge was approximated by a polynomial $\lambda(s)=A s+B s^{3}$ that takes into account the odd symmetry $\lambda(-s)=-\lambda(s)$, where $A$ and $B$ are the constants to be determined by the Galerkin method. Finally, the torque on the particle is computed as

$$
\tau_{z}=\int_{-a}^{a} s \lambda(s) E_{0 y} d s .
$$

Figure 10 shows the electrical torque on the nanowire $\tau_{\text {wall }}$ normalized with the torque far from the wall $\tau_{\text {bulk }}$ as a function of nondimensional distance to the wall $h / b$ computed by using finite elements and using the slender-body approximation. The results are almost coincident and show that the electrical torque near the wall can be reduced up to a factorof 0.6 with respect to the electrical torque in the bulk.
[1] Y. Cui, Q. Wei, H. Park, and C. M. Lieber, Science 293, 1289 (2001).

[2] Y. Li, F. Qian, J. Xiang, and C. M. Lieber, Materials Today 9 , 18 (2006).

[3] M. Law, L. E. Greene, J. C. Johnson, R. Saykally, and P. Yang, Nat. Mater. 4, 455 (2005).
[4] J. J. Boote and S. D. Evans, Nanotechnology 16, 1500 (2005).

[5] B. C. Gierhart, D. G. Howitt, S. J. Chen, R. L. Smith, and S. D. Collins, Langmuir 23, 12450 (2007).

[6] L. Zhang and Y. Zhu, Appl. Phys. Lett. 96, 141902 (2010).

[7] D. L. Fan, F. Q. Zhu, X. Xu, R. C. Cammarata, and C. L. Chien, Proc. Natl. Acad. Sci. 109, 9309 (2012). 
[8] Y. Ren, D. Morganti, H. Y. Jiang, A. Ramos, and H. Morgan, Langmuir 27, 2128 (2011).

[9] P. García-Sánchez, Y. Ren, J. J. Arcenegui, H. Morgan, and A. Ramos, Langmuir 28, 13861 (2012).

[10] J. J. Arcenegui, A. Ramos, P. Garcia-Sanchez, and H. Morgan, Electrophoresis 34, 979 (2013).

[11] J. J. Arcenegui, P. García-Sánchez, H. Morgan, and A. Ramos, Phys. Rev. E 88, 033025 (2013).

[12] J. J. Arcenegui, P. García-Sánchez, H. Morgan, and A. Ramos, Phys. Rev. E 88, 063018 (2013).

[13] T. B. Jones, Electromechanics of Particles (Cambridge University Press, Cambridge, England, 2005).

[14] H. Morgan and N. G. Green, AC Electrokinetics: Colloids and Nanoparticles (Research Studies Press Ltd., Baldock, England, 2003).

[15] K. A. Rose, J. A. Meier, G. M. Dougherty, and J. G. Santiago, Phys. Rev. E 75, 011503 (2007).

[16] T. M. Squires and M. Z. Bazant, J. Fluid Mech. 560, 65 (2006).

[17] D. Saintillan, E. Darve, and E. S. G. Shaqfeh, J. Fluid Mech. 563, 223 (2006).

[18] K. A. Rose, B. Hoffman, D. Saintillan, E. S. G. Shaqfeh, and J. G. Santiago, Phys. Rev. E 79, 011402 (2009).

[19] M. Z. Bazant and T. M. Squires, Curr. Opin. Colloid Interface Sci. 15, 203 (2010).

[20] N. G. Green, A. Ramos, A. González, H. Morgan, and A. Castellanos, Phys. Rev. E 66, 026305 (2002).

[21] A. J. Pascall and T. M Squires, Phys. Rev. Lett. 104, 088301 (2010).

[22] M. Z. Bazant, M. S. Kilic, B. D. Storey, and A. Ajdari, Adv. Colloid Interface Sci. 152, 48 (2009).
[23] Y. K. Suh and S. Kang, Phys. Rev. E 79, 046309 (2009).

[24] R. J. Messinger and T. M. Squires, Phys. Rev. Lett. 105, 144503 (2010).

[25] O. Schnitzer and E. Yariv, Phys. Rev. E 86, 061506 (2012).

[26] S. M. Davidson, M. B. Andersen, and A. Mani, Phys. Rev. Lett. 112, 128302 (2014).

[27] G. Li and J. X. Tang, Phys. Rev. E 69, 061921 (2004).

[28] Y. Han, A. M. Alsayed, M. Nobili, J. Zhang, T. C. Lubensky, and A. G. Yodh, Science 314, 626 (2006).

[29] Y. K. Chen and C. P. Yu, Aerosol Sci. Technol. 16, 255 (1992).

[30] N. B. Viana, R. T. S. Freire, and O. N. Mesquita, Phys. Rev. E 65, 041921 (2002).

[31] M. C. Wang and G. E. Uhlenbeck, Rev. Mod. Phys. 17, 323 (1945).

[32] S. Lobo, C. Escauriaza, and A. Celedon, Langmuir 27, 2142 (2011).

[33] N. G. Green, A. Ramos, A. González, H. Morgan, and A. Castellanos, Phys. Rev. E 61, 4011 (2000).

[34] V. Studer, A. Pépin, Y. Chen, and A. Ajdari, Analyst 129, 944 (2004).

[35] M. Mercedes Tirado, C. Lopez Martinez, and J. Garcia de la Torre, J. Chem. Phys. 81, 2047 (1984).

[36] S. Broersma, J. Chem. Phys. 74, 6989 (1981).

[37] H. A. Stone, J. R. Lister, and M. P. Brenner, Proc. R. Soc. A 455, 329 (1999).

[38] Moses M. Hohman, Michael Shin, Gregory Rutledge, and Michael P. Brenner, Phys. Fluids 13, 2201 (2001).

[39] E. J. Hinch, Perturbation Methods (Cambridge University Press, Cambridge, England, 1991). 